\title{
25 Research Soure \\ Prevalence and Management of Constipation and GI Diagnoses in Children With Solid Tumors
}

\section{Jennifer Belsky ( $\boldsymbol{\sim}$ jbelsky@iu.edu )}

Riley Hospital for Children: Riley Hospital for Children at Indiana University Health https://orcid.org/00000002-2260-0395

\section{Joseph R. Stanek}

Nationwide Children's Hospital Department of Pediatric Hematology Oncology and Bone Marrow

Transplants: Nationwide Children's Hospital Hematology Oncology \& Blood and Marrow Transplant

Nicholas D. Yeager

Nationwide Children's Hospital Hematology Oncology \& Blood and Marrow Transplant

\section{Daniel V. Runco}

Riley Hospital for Children at Indiana University Health

\section{Research Article}

Keywords: Pediatric, oncology, osteopathic, integrative medicine

Posted Date: September 13th, 2021

DOI: https://doi.org/10.21203/rs.3.rs-800140/v1

License: (a) (i) This work is licensed under a Creative Commons Attribution 4.0 International License. Read Full License 


\section{Abstract \\ Purpose}

Despite continued development of targeted therapies for children with cancer, patients continue to experience an array of unwanted side effects. Children with solid tumors may experience constipation as a result many treatment variables. Our objective was to investigate the prevalence and treatment of constipation in hospitalized children with solid tumors treated with chemotherapy.

\section{Methods}

We retrospectively analyzed data from 48 children's hospitals in the Pediatric Health Information System, extracting patients 0-21 years of age with a solid tumor diagnosis hospitalized from October 2015-December 2019. Primary study outcome investigated which solid tumor subgroups received the diagnosis of constipation or received the most constipation medications while receiving chemotherapy for a cancer diagnosis.

\section{Results}

We identified 13,375 unique patients with a solid tumor diagnosis receiving chemotherapy. Constipation was the most common gastrointestinal complaint with 8,658 (64.7\%; 95\% Cl: 63.9-65.5\%) meeting our defined constipation diagnosis. Bone cancers had the highest percentage $(69.9 \%)$ of patients with constipation, while Hodgkin's lymphoma had the lowest, though $52.1 \%$ of patients were affected. A total of $44 \%(n=35,301)$ of encounters received an opioid at some point during admission. Of patients receiving constipation medications, the most commonly prescribed was poly-ethyl glycol $(n=25,175,31.7 \%)$, followed by docusate $(n=11,297,14.2 \%)$, senna $(n=10,325,13.0 \%)$, and lactulose $(n=5,501,6.9 \%)$.

\section{Conclusions}

Constipation is the most common gastrointestinal issue that children with solid tumors experience while receiving chemotherapy. Increased attention should be given to constipation prophylaxis and treatment in children with solid tumors undergoing chemotherapy.

\section{Introduction}

Childhood cancer remains the second leading cause of death in children aged 5 to 14 years of age[1]. The most common diagnoses in the United States (US) include leukemias, central nervous system (CNS) tumors, and lymphomas along with a variety of other solid tumors[2]. Children with cancer undergoing treatment suffer a litany of unwanted side effects during and after their therapy. While survival rates continue to improve with the incorporation of advance immuno- and targeted therapy, many CNS and non-CNS solid tumor treatments continue to rely on traditional cytotoxic and radiotherapy-based treatment agents. Chemotherapy 
induced constipation (CIC) has been well-studied in the adult oncology literature and is the third most common unwanted side effect in patients receiving cytotoxic chemotherapy, with $50-87 \%$ of patients experiencing CIC[3]. Vinca alkaloids are a common cause of constipation, with $80-90 \%$ of adult oncology patients receiving them reporting $\mathrm{CIC}[4]$. While data exists for constipation in the general pediatric population, no studies have explored constipation burden or sequela in children with solid tumors. In addition, literature has not investigated the use of preemptive constipation management during treatment for children receiving chemotherapy.

Constipation accounts for $3 \%$ of general pediatric outpatient visits and $25 \%$ of pediatric gastrointestinal (GI) specialist visits in the United States[5]. Children with constipation suffer from an array of physical symptoms including abdominal pain, cramping, fecal incontinence, rectal fissures, enuresis, and urinary tract infections[6]. In children without cancer, functional constipation has an increased healthcare burden compared to children without constipation. Treatment can be challenging in otherwise healthy children but creates unique challenges for the child undergoing cancer treatment. Constipation management utilizes both pharmacologic and nonpharmacologic interventions to improve symptoms[4]. Nonpharmacologic interventions, such as increased activity and hydration, may be difficult for children with cancer to adhere to due to nausea, mucositis, anorexia, fatigue, or other treatment effects. Despite ongoing advancements in the pediatric oncology field, there remains a lack of guidance for oncology teams with regard to constipation management.

Literature is bereft of studies investigating the incidence and management of constipation in pediatric oncology patients. A prospective questionnaire from 2011 estimated 57-77\% of children requiring chemotherapy treatment for an oncology diagnosis experienced constipation, as defined by the North American Society of Pediatric Gastroenterology, Hepatology, and Nutrition Criteria (NASPGHAN)[7]. Constipation is the most common GI diagnosis during acute lymphoblastic leukemia (ALL) induction therapy affecting $34 \%$ of children, and demonstrating a higher prevalence in females, those with extended hospital stays, and patients receiving opioids. In addition, a wide variety of constipation medications were identified, with $81 \%$ of patients receiving at least one laxative during induction[8]. Most children receiving chemotherapy for a solid tumor diagnosis undergo multiple extended hospital admissions, may require surgical resections impairing mobility, and often receive adjunct radiation therapy. In addition, side effects including mucositis resulting in dehydration and pain control can predispose patients to constipation.

Despite ongoing advancements in the pediatric oncology field, there remains a lack of guidance with regard to constipation management. The objective of our study was to utilize a national administrative database to describe the prevalence of constipation, GI diagnoses, variability of inpatient management, and investigate potential risk factors associated with constipation during hospitalizations for pediatric patients with solid tumors in the United States.

\section{Methods}

\section{Data Source}


Data for this retrospective multicenter cohort study were obtained from the Pediatric Hospital Information System (PHIS) database. Managed by the Children's Hospital Association (CHA) (Overland Park, Kansas), the PHIS database provides detailed information about hospital-based discharges from 48 of the largest freestanding children's hospitals across the US. The participating institutions represent all US census geographic regions and the majority of US tertiary care pediatric hospitals. Reliability and validity are continuously assured by data quality assessments from both $\mathrm{CHA}$ and participating institutions. Patient data are deidentified and given a unique patient identification number, thus allowing patients to be tracked over time and across multiple admissions.

\section{Study Population}

Our study population included patients aged 0 to 21 years with a solid tumor diagnosis admitted to the hospital between October 2015 and December 2019. To ensure patients were receiving chemotherapy for an active solid tumor malignancy, patients were required to have an ICD-10-CM diagnosis code for a solid tumor and a billing code for a central line supply code, chemotherapy administration procedural code, or a chemotherapy medication code at any point during the study time period. Solid cancers of interest were grouped by organ system and included CNS, bone, liver/biliary, kidney, retinal, nonspecific abdominal/pelvic, nonspecific adrenal tumors, and the remaining diagnoses were classified as other solid tumors. Hodgkin's and non-Hodgkin's lymphomas (NHL) were also identified (supplementary 1). Patients with cancer diagnoses in multiple cancer groups were excluded.

\section{Study Definitions}

Previously published methods were utilized for consistency[8]. ICD-10-CM codes were used to identify diagnoses of constipation (K59.XX) and other GI diagnoses, such as appendicitis, gastritis, and ulcers; and GI symptoms, such as nausea and abdominal pain (supplemental 1). Billing codes were used to identify the receipt of chemotherapy agents, opioids, and constipation medications, as well as operating room (OR) and total parenteral nutrition (TPN) charges. Patients who lacked a diagnosis code of constipation but received at least two unique constipation medications were also defined as a case of constipation. Dates of medication administration were extracted to calculate the start and duration of medication use. To evaluate the possible effect of opioid use on the risk of constipation during the admission, we categorized patients into four groups to best distinguish opioid use between anesthesia and pain: 1) no occurrences of opioid use, 2) patient received fentanyl only, 3) patient received other, non-fentanyl opioids for 1 or 2 days, and 4) patient received $\geq$ 2 days of other, non-fentanyl opioids.

Demographic information, such as patient sex, race, and geographic region, and hospitalization information, including as length of stay, intensive care utilization, and mortality, was also obtained from the PHIS database. Patient age was calculated as the age at their last encounter during the study period.

\section{Statistical Analysis:}

All data were summarized using descriptive statistics. Median and range were used to describe quantitative variables and frequency and percentage were used for qualitative variables. The prevalence of constipation among all solid cancers (as well as in specific cancer groups) was calculated as a percentage. The management of constipation was summarized descriptively. Univariate comparisons between those with and 
without constipation were performed using chi-square tests for qualitative variables and Wilcoxon rank sum tests for quantitative variables. Statistical significance was determined by $p$-value $<0.05$. Statistical analyses were performed using Statistical Analysis System software 9.4 (SAS Institute, Cary, NC).

\section{Results:}

\section{Demographics}

We identified 13,375 unique patients ( 79,530 unique admissions) who were admitted with a solid tumor diagnosis from 48 PHIS hospitals during the four-year period evaluated (Table 1). The majority of patients were male $(n=7,465,55.8 \%)$ with a median age of 9.9 years (range: $0.0-20.9$ years). CNS cancers were the most commonly identified solid tumor group (24.4\%), followed by NHL (14.4\%), bone (12.9\%), Hodgkin lymphoma (10.2\%), kidney (8.3\%), abdomen/pelvis (6.8\%), adrenal (6.7\%), liver/biliary (5.1\%), and retinal (2.8\%). Solid tumors of other organ systems not specified here accounted for $8.3 \%$ of the cohort (Supplemental 1). 
Table 1

Demographics and clinical characteristics of pediatric patients with solid tumors (Pediatric Hospital Information System, 2015-2019)

\begin{tabular}{|c|c|}
\hline Characteristic & $\mathbf{N}(\%)$ \\
\hline Unique Patients & 13,375 \\
\hline Male sex & $7,465(55.8)$ \\
\hline \multicolumn{2}{|l|}{ Race } \\
\hline White & $8,550(63.9)$ \\
\hline Black & $1,757(13.1)$ \\
\hline Asian & $605(4.5)$ \\
\hline Other/Unknown & $2,463(18.4)$ \\
\hline Age at last encounter (years) & 9.9 (infant - 21) \\
\hline \multicolumn{2}{|l|}{ Solid cancer diagnosis } \\
\hline CNS & $3,264(24.4)$ \\
\hline Bone & $1,730(12.9)$ \\
\hline \multicolumn{2}{|l|}{ Lymphoma } \\
\hline Hodgkin & $1,365(10.2)$ \\
\hline Non-Hodgkin & $1,925(14.4)$ \\
\hline Liver/Biliary & $686(5.1)$ \\
\hline Kidney & $1,111(8.3)$ \\
\hline Retinal & $378(2.8)$ \\
\hline Abdominal/pelvic NOS & $910(6.8)$ \\
\hline Adrenal tumors NOS & $896(6.7)$ \\
\hline Other solid tumors ${ }^{1}$ & $1,110(8.3)$ \\
\hline \multicolumn{2}{|c|}{${ }^{1}$ Includes other solid tumors/masses of other organ systems no listed } \\
\hline
\end{tabular}

\section{Prevalence of Constipation and GI Diagnoses}

Constipation was the most common GI complaint identified in unique solid tumor patients, with 8,658 (64.7\%) being diagnosed with constipation or receiving at least two constipation medications during a single admission (Table 2). Bone cancers had the highest prevalence (79.7\%) of patients with constipation, while retinal tumors had the lowest at 23.3\% (Fig. 1). Nausea/vomiting ( $n=5,439,48.6 \%)$ and abdominal pain ( $n=$ $1,044,9.3 \%$ ) were the next most commonly observed GI diagnoses. Other GI symptoms commonly reported in 
solid cancer patients included mucositis $(n=3,017,26.9 \%)$ and gastroesophageal reflux disease $(n=1,419$, $12.7 \%)$. Mucositis was most common in patients with NHL (42.7\%), bone cancers (41.7), and adrenal tumors (38.5\%), and least commonly seen in kidney $(10.4 \%)$ and abdominal/pelvic (10.2\%) tumors. Gl infections were present in all diagnoses, with adrenal tumors $(n=195,21.8 \%)$ having the most, and Hodgkin lymphoma patients $(n=101,7.4 \%)$ with the fewest reported Gl infections. 
Table 2

Patient prevalence of most commonly identified solid tumor diagnoses with various GI diagnoses (Pediatric Hospital Information System, 2015-2019)

\begin{tabular}{|c|c|c|c|c|c|c|c|c|}
\hline Diagnosis & $\begin{array}{l}\text { All } \\
\text { Solid } \\
\text { Tumors } \\
\mathrm{N}(\%)\end{array}$ & $\begin{array}{l}\text { CNS } \\
\text { Cancers } \\
\text { N (\%) }\end{array}$ & $\begin{array}{l}\text { Bone } \\
\text { Cancers } \\
\text { N (\%) }\end{array}$ & $\begin{array}{l}\text { Kidney } \\
N(\%)\end{array}$ & $\begin{array}{l}\text { Abd/Pelvic } \\
N(\%)\end{array}$ & $\begin{array}{l}\text { Adrenal } \\
\text { tumors } \\
\mathrm{N}(\%)\end{array}$ & $\begin{array}{l}\mathrm{HL} \\
\mathrm{N}(\%)\end{array}$ & $\begin{array}{l}\text { NHL } \\
\text { N (\%) }\end{array}$ \\
\hline Total Patients & & 3,264 & 1,730 & 1,111 & 910 & 896 & 1,365 & 1,925 \\
\hline $\begin{array}{l}\text { Defined } \\
\text { Constipation } 1\end{array}$ & $\begin{array}{l}8,658 \\
(64.7)\end{array}$ & $\begin{array}{l}2,281 \\
(69.9)\end{array}$ & $\begin{array}{l}1,379 \\
(79.7)\end{array}$ & $\begin{array}{l}734 \\
(66.1)\end{array}$ & $547(60.1)$ & $\begin{array}{l}508 \\
(56.7)\end{array}$ & $\begin{array}{l}711 \\
(52.1)\end{array}$ & $\begin{array}{l}1,307 \\
(67.9)\end{array}$ \\
\hline $\begin{array}{l}\text { Constipation (Dx } \\
\text { only) }\end{array}$ & $\begin{array}{l}6,477 \\
(48.4)\end{array}$ & $\begin{array}{l}1,583 \\
(48.5)\end{array}$ & $\begin{array}{l}1,143 \\
(66.1)\end{array}$ & $\begin{array}{l}515 \\
(46.4)\end{array}$ & $419(46.0)$ & $\begin{array}{l}422 \\
(47.1)\end{array}$ & $\begin{array}{l}536 \\
(39.3)\end{array}$ & $\begin{array}{l}984 \\
(51.1)\end{array}$ \\
\hline \multicolumn{9}{|l|}{ GI Symptoms } \\
\hline Abdominal Pain & $\begin{array}{l}1,185 \\
(8.9)\end{array}$ & $\begin{array}{l}255 \\
(7.8)\end{array}$ & $\begin{array}{l}182 \\
(10.5)\end{array}$ & $\begin{array}{l}86 \\
(7.7)\end{array}$ & $86(9.5)$ & $\begin{array}{l}101 \\
(11.3)\end{array}$ & $\begin{array}{l}104 \\
(7.6)\end{array}$ & $\begin{array}{l}230 \\
(12.0)\end{array}$ \\
\hline Nausea/Vomiting & $\begin{array}{l}6,423 \\
(48.0)\end{array}$ & $\begin{array}{l}1,440 \\
(44.1)\end{array}$ & $\begin{array}{l}1,204 \\
(69.6)\end{array}$ & $\begin{array}{l}367 \\
(33.0)\end{array}$ & $412(45.3)$ & $\begin{array}{l}445 \\
(49.7)\end{array}$ & $\begin{array}{l}646 \\
(47.3)\end{array}$ & $\begin{array}{l}925 \\
(48.1)\end{array}$ \\
\hline \multicolumn{9}{|l|}{$\begin{array}{l}\text { Other GI } \\
\text { Diagnoses }\end{array}$} \\
\hline GERD & $\begin{array}{l}1,587 \\
(11.9)\end{array}$ & $\begin{array}{l}415 \\
(12.7)\end{array}$ & $\begin{array}{l}271 \\
(15.7)\end{array}$ & $\begin{array}{l}66 \\
(5.9)\end{array}$ & $90(9.9)$ & $\begin{array}{l}103 \\
(11.5)\end{array}$ & $\begin{array}{l}121 \\
(8.9)\end{array}$ & $\begin{array}{l}254 \\
(13.2)\end{array}$ \\
\hline Ulcer & $\begin{array}{l}96 \\
(0.7)\end{array}$ & $29(0.9)$ & $9(0.5)$ & $0(-)$ & $10(1.1)$ & $4(0.5)$ & $\begin{array}{l}8 \\
(0.6)\end{array}$ & $\begin{array}{l}27 \\
(1.4)\end{array}$ \\
\hline Gastritis & $\begin{array}{l}1,568 \\
(11.7)\end{array}$ & $\begin{array}{l}302 \\
(9.3)\end{array}$ & $\begin{array}{l}232 \\
(13.4)\end{array}$ & $\begin{array}{l}75 \\
(6.8)\end{array}$ & $86(9.5)$ & $\begin{array}{l}163 \\
(18.2)\end{array}$ & $\begin{array}{l}165 \\
(12.1)\end{array}$ & $\begin{array}{l}319 \\
(16.6)\end{array}$ \\
\hline Appendicitis & $\begin{array}{l}221 \\
(1.7)\end{array}$ & $36(1.1)$ & $32(1.9)$ & $\begin{array}{l}11 \\
(1.0)\end{array}$ & $6(0.7)$ & $\begin{array}{l}22 \\
(2.5)\end{array}$ & $\begin{array}{l}24 \\
(1.8)\end{array}$ & $\begin{array}{l}68 \\
(3.5)\end{array}$ \\
\hline IBD & $\begin{array}{l}62 \\
(0.5)\end{array}$ & $8(0.3)$ & $5(0.3)$ & $4(0.4)$ & $4(0.4)$ & $2(0.2)$ & $\begin{array}{l}8 \\
(0.6)\end{array}$ & $\begin{array}{l}26 \\
(1.4)\end{array}$ \\
\hline IBS & $\begin{array}{l}25 \\
(0.2)\end{array}$ & $6(0.2)$ & $8(0.5)$ & $0(-)$ & $3(0.3)$ & $0(-)$ & $\begin{array}{l}1(< \\
0.1)\end{array}$ & $\begin{array}{l}7 \\
(0.4)\end{array}$ \\
\hline NEC & $\begin{array}{l}6(< \\
0.1)\end{array}$ & $2(<0.1)$ & $0(-)$ & $0(-)$ & $0(-)$ & $1(0.1)$ & $\begin{array}{l}1(< \\
0.1)\end{array}$ & $0(-)$ \\
\hline Pancreatic Issues & $\begin{array}{l}245 \\
(1.8)\end{array}$ & $31(1.0)$ & $12(0.7)$ & $\begin{array}{l}12 \\
(1.1)\end{array}$ & $32(3.5)$ & $\begin{array}{l}12 \\
(1.3)\end{array}$ & $\begin{array}{l}8 \\
(0.6)\end{array}$ & $\begin{array}{l}115 \\
(6.0)\end{array}$ \\
\hline
\end{tabular}

\footnotetext{
${ }^{1}$ Administration of constipation medications without the presence of a constipation diagnosis.

Abbreviations: $\mathrm{CNS}=$ Central Nervous System, Abd = Abdominal, $\mathrm{HL}=$ Hodgkin Lymphoma, NHL (NonHodgkin Lymphoma), GERD = Gastroesophageal Reflux Disease, IBD = Irritable Bowel Disease, IBS = Irritable Bowel Syndrome
} 


\begin{tabular}{|c|c|c|c|c|c|c|c|c|}
\hline Diagnosis & $\begin{array}{l}\text { All } \\
\text { Solid } \\
\text { Tumors } \\
\mathrm{N}(\%)\end{array}$ & $\begin{array}{l}\text { CNS } \\
\text { Cancers } \\
\text { N (\%) }\end{array}$ & $\begin{array}{l}\text { Bone } \\
\text { Cancers } \\
\text { N (\%) }\end{array}$ & $\begin{array}{l}\text { Kidney } \\
N(\%)\end{array}$ & $\begin{array}{l}\text { Abd/Pelvic } \\
N(\%)\end{array}$ & $\begin{array}{l}\begin{array}{l}\text { Adrenal } \\
\text { tumors }\end{array} \\
\mathrm{N}(\%)\end{array}$ & $\begin{array}{l}\mathrm{HL} \\
\mathrm{N}(\%)\end{array}$ & $\begin{array}{l}\mathrm{NHL} \\
\mathrm{N}(\%)\end{array}$ \\
\hline $\begin{array}{l}\text { Gallbladder } \\
\text { Issues }\end{array}$ & $\begin{array}{l}237 \\
(1.8)\end{array}$ & $11(0.3)$ & $11(0.6)$ & $9(0.8)$ & $29(3.2)$ & $\begin{array}{l}14 \\
(1.6)\end{array}$ & $\begin{array}{l}11 \\
(0.8)\end{array}$ & $\begin{array}{l}51 \\
(2.7)\end{array}$ \\
\hline $\begin{array}{l}\text { Anal/Rectal } \\
\text { Issues }\end{array}$ & $\begin{array}{l}751 \\
(5.6)\end{array}$ & $\begin{array}{l}119 \\
(3.7)\end{array}$ & $\begin{array}{l}254 \\
(14.7)\end{array}$ & $\begin{array}{l}25 \\
(2.3)\end{array}$ & $39(4.3)$ & $\begin{array}{l}37 \\
(4.1)\end{array}$ & $\begin{array}{l}56 \\
(4.1)\end{array}$ & $\begin{array}{l}149 \\
(7.7)\end{array}$ \\
\hline Peritonitis & $\begin{array}{l}362 \\
(2.7)\end{array}$ & $30(0.9)$ & $6(0.4)$ & $\begin{array}{l}78 \\
(7.0)\end{array}$ & $64(7.0)$ & $\begin{array}{l}29 \\
(3.2)\end{array}$ & $\begin{array}{l}3 \\
(0.2)\end{array}$ & $\begin{array}{l}55 \\
(2.9)\end{array}$ \\
\hline Mucositis & $\begin{array}{l}3,436 \\
(25.7)\end{array}$ & $\begin{array}{l}551 \\
(16.9)\end{array}$ & $\begin{array}{l}722 \\
(41.7)\end{array}$ & $\begin{array}{l}116 \\
(10.4)\end{array}$ & $93(10.2)$ & $\begin{array}{l}345 \\
(38.5)\end{array}$ & $\begin{array}{l}369 \\
(27.0)\end{array}$ & $\begin{array}{l}821 \\
(42.7)\end{array}$ \\
\hline GI Infection & $\begin{array}{l}1,650 \\
(12.3)\end{array}$ & $\begin{array}{l}414 \\
(12.7)\end{array}$ & $\begin{array}{l}210 \\
(12.1)\end{array}$ & $\begin{array}{l}118 \\
(10.6)\end{array}$ & $76(8.4)$ & $\begin{array}{l}195 \\
(21.8)\end{array}$ & $\begin{array}{l}101 \\
(7.4)\end{array}$ & $\begin{array}{l}305 \\
(15.8)\end{array}$ \\
\hline \multicolumn{9}{|c|}{${ }^{1}$ Administration of constipation medications without the presence of a constipation diagnosis. } \\
\hline \multicolumn{9}{|c|}{$\begin{array}{l}\text { Abbreviations: CNS = Central Nervous System, Abd = Abdominal, } \mathrm{HL}=\text { Hodgkin Lymphoma, NHL (Non- } \\
\text { Hodgkin Lymphoma), GERD = Gastroesophageal Reflux Disease, IBD = Irritable Bowel Disease, IBS = } \\
\text { Irritable Bowel Syndrome }\end{array}$} \\
\hline
\end{tabular}

\section{Characteristics of Constipation Admissions}

Inpatient admissions with constipation were more likely to be female $(n=23,420,57.3 \%)$ and/or utilize a narcotic $(n=14,044,56.8 \%)$ (Table 3$)$. In addition, admissions with constipation were more likely to have OR charges $(56.8 \%$ vs $39.9 \% ; p<.0001)$ and TPN use $(10.5 \%$ vs $6.7 \% ; p<.0001)$ compared to admissions without constipation. 
Table 3

Characteristics of pediatric patients with solid tumor admissions with constipation

\begin{tabular}{|llll|}
\hline & $\begin{array}{l}\text { Constipation admits } \\
\mathbf{N}=\mathbf{2 4 , 7 1 9}\end{array}$ & $\begin{array}{l}\text { No constipation } \\
\mathbf{N}=54,811\end{array}$ & p-value \\
\hline Median age (IQR) & $10.6(5.0-15.5)$ & $10.1(4.1-15.1)$ & $<.0001$ \\
\hline Male sex & $13,358(54.0)$ & $31,391(57.3)$ & $<.0001$ \\
\hline Race & & & $<.0001$ \\
\hline White & $15,991(64.7)$ & $35,070(64.0)$ & \\
\hline Black & $3,180(12.9)$ & $6,678(12.2)$ & \\
\hline Asian & $1,157(4.7)$ & $2,635(4.8)$ & \\
\hline Other & $4,391(17.8)$ & $10,428(19.0)$ & \\
\hline Ethnicity & & & $<.0001$ \\
\hline Hispanic/Latino & $5,263(21.3)$ & $11,504(21.0)$ & \\
\hline Non-Hispanic/Latino & $18,284(74.0)$ & $40,254(73.4)$ & \\
\hline Other/Unknown & $1,172(4.7)$ & $3,053(5.6)$ & \\
\hline Median LOS (IQR) & $5(3-10)$ & $3(2-5)$ & $<.0001$ \\
\hline Insurance type & & & \\
\hline Public & $11,347(45.9)$ & $25,177(45.9)$ & \\
\hline Private & $11,912(48.2)$ & $26,165(47.7)$ & \\
\hline Other & $882(3.6)$ & $2,293(4.2)$ & \\
\hline Unknown & $578(2.3)$ & $1,176(2.2)$ & \\
\hline Use of narcotic & $14,044(56.8)$ & $21,859(39.9)$ & $<.0001$ \\
\hline OR charges & $7,236(29.3)$ & $10,240(18.7)$ & $<.0001$ \\
\hline TPN & $2,592(10.5)$ & $3,661(6.7)$ & \\
\hline Abbreviations: OR = operating room, TPN = total parental nutrition & \\
\hline
\end{tabular}

\section{Constipation Management and Opioid Use}

A constipation medication was administered in $45.8 \%(n=36,444)$ of admissions (Table 4$)$. These medications were used in the setting of a constipation diagnosis in $73.1 \%(n=11,912)$ of admissions, while $38.8 \%(n=24,532)$ of admissions utilized a constipation medication without the presence of a constipation diagnosis code. Among admissions with a constipation diagnosis, $21.9 \%(n=3,568)$ required 2 different 
constipation medications, with $18.2 \%$ of admissions requiring 3 or more unique constipation medications. In admissions without the presence of a constipation diagnosis, a single agent was used $24.1 \%$ of the time, whereas $13.3 \%(n=8,413)$ received a combination of 2 or more different medications. The most commonly utilized constipation medication, regardless of the presence of a constipation diagnosis, was polyethyl glycol $(n=25,175,31.7 \%)$, followed by docusate $(n=11,297,14.2 \%)$, senna $(n=10,325,13.0 \%)$, and lactulose $(n=$ $5,501,6.9 \%)$. These medications were used for a median of 2 to 3 days. A total of $45 \%(n=35,903)$ of encounters received an opioid at some point during an admission: $4.5 \%(n=3,598)$ received fentanyl only, $15.2 \%(12,068)$ received $\leq 2$ days of a non-fentanyl opioid, and $25.4 \%(n=20,237)$ received $>2$ days of a nonfentanyl opioid. The extended use of non-fentanyl opioids ( $>2$ days) was more common in admissions with a constipation diagnosis compared to those without a constipation diagnosis $(33.1 \%$ vs $23.5 \%$; $p<.0001)$.

Constipation management and opioid use in specific solid tumor diagnoses are detailed in supplemental 2. 
Table 4

Constipation medical management and opioid utilization in pediatric patients with solid tumors during inpatient admission (Pediatric Hospital Information System, 2015-2019).

\begin{tabular}{|c|c|c|c|}
\hline \multirow[t]{2}{*}{ Medication } & $\begin{array}{l}\text { All } \\
\text { Admissions }\end{array}$ & $\begin{array}{l}\text { Constipation } \\
\text { Admissions }\end{array}$ & $\begin{array}{l}\text { No Constipation } \\
\text { Admissions }^{1}\end{array}$ \\
\hline & $N=79,530$ & $N=16,306$ & $N=63,224$ \\
\hline Anti-Constipation & $\begin{array}{l}36,444 \\
(45.8)\end{array}$ & $11,912(73.1)$ & $24,532(38.8)$ \\
\hline Polyethyl Glycol-electrolyte & $\begin{array}{l}25,175 \\
(31.7)\end{array}$ & $8,874(54.4)$ & $16,301(25.8)$ \\
\hline Senna & $\begin{array}{l}10,325 \\
(13.0)\end{array}$ & $4,384(26.9)$ & $5,941(9.4)$ \\
\hline Lactulose & $5,501(6.9)$ & $2,272(16.7)$ & $2,774(4.4)$ \\
\hline Docusate & $\begin{array}{l}11,297 \\
(14.2)\end{array}$ & $4,139(25.4)$ & $7,158(11.3)$ \\
\hline Electrolyte Laxatives & $865(1.1)$ & $559(3.4)$ & $306(0.5)$ \\
\hline Glycerin & $1,745(2.2)$ & $641(3.9)$ & $1,104(1.7)$ \\
\hline Mineral Oil & $834(1.0)$ & $312(1.9)$ & $522(0.8)$ \\
\hline Bisacodyl & $2,094(2.6)$ & $888(5.4)$ & $1,206(1.9)$ \\
\hline Laxative Combination $^{2}$ & $806(1.0)$ & $400(2.5)$ & $406(0.6)$ \\
\hline \multicolumn{4}{|c|}{ Total Different Laxatives Taken } \\
\hline 0 & $\begin{array}{l}44,229 \\
(55.6)\end{array}$ & $4,679(28.7)$ & $39,550(62.6)$ \\
\hline 1 & $\begin{array}{l}20,359 \\
(25.6)\end{array}$ & $5,098(31.3)$ & $15,261(24.1)$ \\
\hline 2 & $9,433(11.9)$ & $3,568(21.9)$ & $5,865(9.3)$ \\
\hline 3 or more & $5,509(6.9)$ & $2,961(18.2)$ & $2,548(4.0)$ \\
\hline \multicolumn{4}{|l|}{ Opioid Group } \\
\hline None & $\begin{array}{l}43,627 \\
(54.9)\end{array}$ & $8,120(49.8)$ & $35,507(56.2)$ \\
\hline Fentanyl Only & $3,598(4.5)$ & $485(3.0)$ & $3,113(4.9)$ \\
\hline$\leq 2$ days of Other Opioids & $\begin{array}{l}12,068 \\
(15.2)\end{array}$ & $2,304(14.1)$ & $9,764(15.4)$ \\
\hline
\end{tabular}

\footnotetext{
${ }^{1}$ Administration of $>2$ constipation medications without the presence of a constipation diagnosis.

${ }^{2}$ Excludes combinations with iron.

Percentages may not sum to $100 \%$ due to rounding.
} 


\begin{tabular}{|llll|}
\hline Medication & $\begin{array}{l}\text { All } \\
\text { Admissions } \\
\mathbf{N}=79,530\end{array}$ & $\begin{array}{l}\text { Constipation } \\
\text { Admissions } \\
\mathbf{N}=16,306\end{array}$ & $\begin{array}{l}\text { No Constipation } \\
\text { Admissions }^{1}\end{array}$ \\
$\mathbf{N}=63,224$
\end{tabular}

\section{Discussion:}

We identified 13,375 unique patients with 79,530 unique admissions in this study of pediatric patients with solid tumors admitted at 48 children's hospitals. A majority of children received constipation medications regardless of having a billed diagnosis or not. The prevalence of constipation identified in our study (64.7\%) is impressively greater than the prevalence reported in the general pediatric population (ranging from $0.7-29 \%[9$, 10]).

For unclear reasons, pediatric females have been demonstrated to have a higher prevalence of constipation than males in healthy children and the pediatric ALL patient population $[8,11]$. Similarly, our data demonstrated that females with solid tumors are at higher risk for experiencing constipation during hospitalization. Additionally, our finding that opioids are associated with constipation has been well studied in adult and pediatric cancer literature[12]. Opioids lead to constipation through their action upon opioid receptors in the $\mathrm{GI}$ tract, leading to reduced $\mathrm{GI}$ propulsion and increased fluid absorption ${ }^{13}$. Unfortunately, opioids are commonly a necessity for cancer related visceral or bone pain. Additionally, patients with solid tumors can undergo surgical interventions for tumor, staging, or central line placement, involving anesthesia and post-procedure pain control. Certain immunotherapies, such as dinutuximab in neuroblastoma patients, may require continuous intravenous pain medications, leading to prolonged opioid use. Non-Hodgkin lymphoma treatment is notoriously intense and can often result in prolonged mucositis resulting in lengthy opioid use for pain control, which likely correlates with the high rates of constipation we identified. Similarly to patients with ALL in induction, we demonstrated a wide variability in constipation medications prescribed, regardless of a constipation diagnosis, further evidence of the lack of standard practice on how to manage constipation in the pediatric oncology setting[8]. 
Previous studies have shown that children with a constipation diagnosis have a significant increase in healthcare utilization compared to children without constipation[13]. Although constipation in healthy children develops insidiously over time and fortunately is almost always secondary to functional constipation, pediatric oncology patients have psychological stressors as a result of their diagnosis and receive chemotherapy and other interventions which predispose them to constipation. While symptoms of constipation go unnoticed or underreported due to patient embarrassment and/or anxiety with the medical team, symptoms and risks of constipation can worsen. Stool withholding, caused by hard, painful bowel movements, anal fissures, and mucositis, can disrupt brain-colonic signaling leading to increased stretch and stool burden, and progressively worsen stool build up[14]. The majority of bowel regimens are readily available and affordable for patients to take in the hospital or at home. In addition, there are minimal to no interactions between constipation medications and cancer directed therapy[15]. Preventative measures and attention to constipation symptoms could eliminate chemotherapy delays and decreases due to severe constipation or chronic constipation habits following chemotherapy. Finally, children with hematologic malignancies and constipation have increased abdominal imaging exposure[8]. Multiple pediatric subspecialist organizations, including the Children's Oncology Group, have previously published that increasing ionizing radiation exposure from $x$-ray and computerized tomography should be avoided as much as possible due to risks of secondary malignant neoplasms[16]. Although in a general pediatric setting, constipation is a clinical diagnosis that rarely requires imaging, children undergoing chemotherapy can have underlying pathophysiology or life-threatening diagnoses such as typhlitis that may necessitate further work up when presenting with abdominal imaging or nausea. Decreasing the physical symptoms of abdominal pain, nausea, and bloating that accompany many patients with constipation, could result in decreased abdominal radiograph exposure and in turn, decrease unnecessary ionizing radiation exposure.

\section{Limitations}

These findings should be interpreted in light of the strengths and limitations of our study design and data source, as outlined in our previous study[8]. These limitations acknowledge that PHIS is a large-scale database that provides multi-institutional, geographically diverse representation of a large number of subjects. Relying on accurate coding and diagnoses using ICD-10 codes is an inherent limitation of PHIS. We cannot assess with certainty how often constipation medications were used to prevent, rather than treat constipation, however, we attempted to account for this conservatively by requiring a diagnosis of constipation before considering the intent to be treatment. It is certainly possible that patients receiving constipation medications for "prevention" by our definition could have been experiencing signs or symptoms of constipation, in which case we have under-estimated the true prevalence of constipation in this patient population. Similarly, in patients who received constipation medications, we are unable to comment on whether the number and type of medication prescribed were appropriate for their needs. In those patients receiving an opioid, we are unable to assess if this was used for sedation/anesthesia purposes or pain control. In an attempt to account for this, we placed patients into four opioid groups of presumed increased constipation risk. We are limited to ICD-10-CM coding to define the specific cancer groups based on location of tumor and have no detail regarding the specific tumor classification, stage, histology, or specific treatment regimen. Finally, the PHIS data set does not allow us to assess the extent of constipation or use of medications after discharge. 


\section{Conclusions}

In summary, constipation is highly prevalent in children with solid tumors receiving chemotherapy, is likely multifactorial in nature, and most children receive a variety of medications to treat it. Clinical practice guidelines and additional supportive care recommendations for constipation are lacking in pediatric oncology. This report demonstrates the high frequency of constipation in pediatric patients with solid tumors and supports the need for increased attention to prophylaxis and management in this population to prevent patient discomfort, minimize potential impact on cancer treatment, as well as reduce exposure to expensive and potentially harmful radiologic testing for evaluation of gastrointestinal symptoms. Supportive care guidelines are sorely needed in this area, particularly for high-risk populations such as solid tumor patients, and future prospective studies should seek to determine the most effective standardized treatment regimens.

\section{Abbreviations}

CNS

Central Nervous System, NOS $=$ Not Otherwise Specified

\section{Declarations}

Funding: The authors did not receive support from any organization for the submitted work.

\section{Conflicts of interest/Competing interests: none}

Availability of data and material: not applicable

Code Availability: not applicable

Authors' Contributions: Drs. Belsky and Runco conceptualized and designed the study, drafted the initial manuscript, and revised the manuscript.

Mr. Stanek designed the data collection instruments, carried out statistical analyses, reviewed and revised the manuscript.

Drs. Yeager and Runco conceptualized the study, reviewed, and revised the manuscript.

Ethics approval: This retrospective chart review study involving human participants was in accordance with the ethical standards of the institutional and national research committee and with the $1964 \mathrm{Helsinki}$ Declaration and its later amendments or comparable ethical standards. The Human Investigation Committee (IRB) of Indiana University approved this study.

Consent to participate: Informed consent was not needed for this retrospective database review

Consent for publication: Consent for publication was not needed for this retrospective review

\section{References}


1. Ward E et al (2014) Childhood and adolescent cancer statistics, 2014. CA Cancer J Clin 64(2):83-103

2. Siegel RL et al., Cancer Statistics, 2021. CA Cancer J Clin, 2021. 71(1): p. 7-33

3. Rhondali W et al (2013) Self-reported constipation in patients with advanced cancer: a preliminary report. J Pain Symptom Manage 45(1):23-32

4. McQuade RM et al (2016) Chemotherapy-Induced Constipation and Diarrhea: Pathophysiology, Current and Emerging Treatments. Front Pharmacol 7:414

5. Sood M, Lichtlen P, Perez MC (2018) Unmet Needs in Pediatric Functional Constipation. Clin Pediatr 57(13):1489-1495

6. Rajindrajith S et al (2016) Childhood constipation as an emerging public health problem. World journal of gastroenterology 22(30):6864-6875

7. Pashankar FD et al (2011) Acute Constipation in Children Receiving Chemotherapy for Cancer. Journal of Pediatric Hematology/Oncology 33(7):e300-e303

8. Belsky JA, Stanek JR, O'Brien SH (2020) Prevalence and management of constipation in pediatric acute lymphoblastic leukemia in U.S. children's hospitals. Pediatr Blood Cancer 67(11):e28659

9. Tabbers MM et al (2014) Evaluation and Treatment of Functional Constipation in Infants and Children: Evidence-Based Recommendations From ESPGHAN and NASPGHAN. J Pediatr Gastroenterol Nutr 58(2):258-274

10. van den Berg MM, Benninga MA, Di Lorenzo C (2006) Epidemiology of childhood constipation: a systematic review. Am J Gastroenterol 101(10):2401-2409

11. Diaz S, Bittar K, Mendez MD, Constipation, in StatPearls. 2020, StatPearls Publishing StatPearls Publishing LLC: Treasure Island (FL)

12. Drewes AM et al (2016) Definition, diagnosis and treatment strategies for opioid-induced bowel dysfunction-Recommendations of the Nordic Working Group. Scand J Pain 11:111-122

13. Liem $O$ et al (2009) Health utilization and cost impact of childhood constipation in the United States. J Pediatr 154(2):258-262

14. Medicine JH (2010) Plugged up: Doctors see signs of worsening constipation in children. Science Daily

15. Schmier JK et al (2014) Cost savings of reduced constipation rates attributed to increased dietary fiber intakes: a decision-analytic model. BMC Public Health 14:374

16. Weiser DA et al (2013) Imaging in childhood cancer: a Society for Pediatric Radiology and Children's Oncology Group Joint Task Force report. Pediatr Blood Cancer 60(8):1253-1260

\section{Figures}




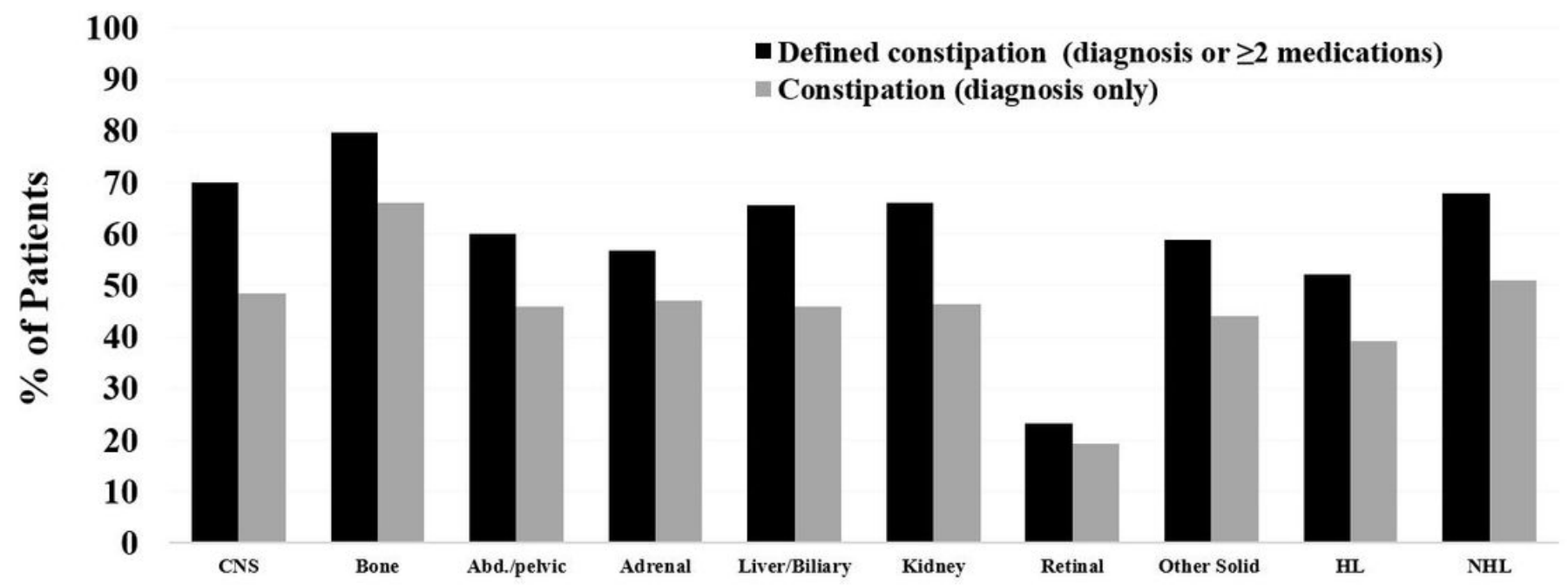

Figure 1

Prevalence of constipation among hospitalized patients with solid cancers (Pediatric Hospital Information System, 2015-2019) Abbreviations: CNS= Central Nervous System, Abd= Abdominal, HL= Hodgkin Lymphoma, NHL= Non-Hodgkin Lymphoma

\section{Supplementary Files}

This is a list of supplementary files associated with this preprint. Click to download.

- Supplementary1and2.docx 